\title{
Towards Noncommutative Linking Numbers via the Seiberg-Witten Map
}

\author{
H. García-Compeán, ${ }^{1}$ O. Obregón, ${ }^{2}$ and R. Santos-Silva ${ }^{2}$ \\ ${ }^{1}$ Departamento de Física, Centro de Investigación y de Estudios Avanzados del IPN, P.O. Box 14-740, 07000 Mexico City, DF, Mexico \\ ${ }^{2}$ Departamento de Física, DCI, Universidad de Guanajuato, 37150 León, GTO, Mexico \\ Correspondence should be addressed to R. Santos-Silva; rsantos@fisica.ugto.mx
}

Received 3 July 2015; Revised 13 September 2015; Accepted 15 September 2015

Academic Editor: Giovanni Amelino-Camelia

Copyright @ 2015 H. García-Compeán et al. This is an open access article distributed under the Creative Commons Attribution License, which permits unrestricted use, distribution, and reproduction in any medium, provided the original work is properly cited.

Some geometric and topological implications of noncommutative Wilson loops are explored via the Seiberg-Witten map. In the abelian Chern-Simons theory on a three-dimensional manifold, it is shown that the effect of noncommutativity is the appearance of $6^{n}$ new knots at the $n$th order of the Seiberg-Witten expansion. These knots are trivial homology cycles which are Poincare dual to the higher-order Seiberg-Witten potentials. Moreover the linking number of a standard 1-cycle with the Poincaré dual of the gauge field is shown to be written as an expansion of the linking number of this 1-cycle with the Poincare dual of the Seiberg-Witten gauge fields. In the process we explicitly compute the noncommutative "Jones-Witten" invariants up to first order in the noncommutative parameter. Finally in order to exhibit a physical example, we apply these ideas explicitly to the Aharonov-Bohm effect. It is explicitly displayed at first order in the noncommutative parameter; we also show the relation to the noncommutative Landau levels.

\section{Introduction}

Noncommutativity of spacetime has strongly attracted the attention in the last two decades (see, e.g., [1, 2]). In a remarkable paper by Seiberg and Witten [3], they showed that open string theory in the specific limit of small volume, nonvanishing $B$-field and $\alpha^{\prime} \rightarrow 0$, the amplitudes with constant open string metric $G$ and noncommutativity parameter $\Theta$, gives precisely a (Connes-style [4]) noncommutative gauge theory. In this context, this theory can be rewritten in terms of the commutative one through a field redefinition known as the Seiberg-Witten map [3].

Under such a map gauge fields are written as an infinite series on the noncommutative parameter $\Theta_{\mu \nu}\left(\left[\widehat{x}_{\mu}, \widehat{x}_{\nu}\right]=\right.$ $\Theta_{\mu \nu}$ where $\widehat{x}_{\mu}$ are the noncommutative coordinates of the spacetime). To every order (in $\Theta$ ) the gauge field can be determined in terms of the usual (commutative) gauge field. The addition of higher-derivative terms does not spoil gauge invariance since the noncommutative gauge group action on the space of noncommutative connections is such that the quotient is isomorphic to the corresponding quotient in the commutative case. This construction was realized explicitly for pure gauge fields. Later this construction was extended to the nonabelian case and coupled to matter fields [5-7]. This proposal has been studied widely in the literature and used for noncommutative gauge invariance extensions of the standard model and gravity (see, e.g., [8-11]). In the case of the gravity such extension gave rise in a natural way to topological invariants such as the Euler characteristic and the signature with explicit computations in $[12,13]$.

It is well known that the Wilson lines and loops are very useful in the description and computation of some nonperturbative aspects of gauge theory just as confinement [14]. In the context of string theory noncommutative Wilson loops have been studied within the correspondence gauge/gravity duality in [15-19]. In [20], in noncommutative gauge theories, Wilson lines were studied through the Schwinger-Dyson equations of correlation functions of Wilson lines. In field theory on a noncommutative two-dimensional torus the correlators of Wilson line operators were determined [21]. Also in the two-dimensional plane within a perturbative expansion (in $\Theta$ and in $1 / \Theta$-expansion), the nonplanar correlation functions of Wilson loops were obtained and the mixing UV/IR was consistently regularized $[22,23]$. Wilson loops 
also were studied in the twisted covariant noncommutative field theory. In particular their correlation functions, Morita duality, and the area-preserving diffeomorphisms action were examined in this context in [24, 25]. In [26], it was observed that, in a gauge theory on the noncommutative plane, the area-preserving diffeomorphisms symmetry is nonperturbatively broken.

Noncommutative gauge theories have very striking topological and geometrical features. For instance, in string theory, in the absence of $B$-field, the instanton equation $F^{+}=$ 0 is exact in $\alpha^{\prime}$ as the instanton shrinks, and this small instanton becomes a singularity of its moduli space. For nonvanishing $B$-field and nonsmall volume, the singularity is resolved and absent from the moduli space. This space defines precisely the moduli space of noncommutative instantons [27]. It is worth mentioning that recently in [28] some geometrical implications of the Seiberg-Witten map in ChernSimons and gravity were discussed. Some useful comments of noncommutative Chern-Simons theory can be found in [29]. Some connections with the Seiberg-Witten equations are described in [30-32]. The consideration of some topological aspects of the noncommutative Wilson lines in the SeibergWitten limit is discussed in [33]. Other aspects of topological nature in noncommutative gauge theory were discussed in [34].

The Wilson loops possess by themselves some interesting geometrical and topological properties; for example, in the abelian case, they can be regarded in terms of the linking number [35]. On the other hand Wilson loops are also useful for the description of knots and link invariants. In [36] Jones polynomials were reproduced and generalized by computing correlation functions of products of Wilson loops using Chern-Simons action in the path integral formalism. Essentially the computations throw topological invariants since the action, observables, and measure do not depend on the metric for Chern-Simons theories and their higherdimensional generalization known as BF theories [37-39]. Linking numbers in BF theories were examined in [37-41].

In the present work we explore some geometrical and topological aspects based on the previous ideas but immersed in a noncommutative space using the Wilson lines constructed by means of the gauge field provided by the SeibergWitten map. As our main result, we will see that in this context it is possible to establish a correspondence between the terms of a power series (in the noncommutative parameter $\Theta$ ) contained within the phase of a noncommutative Wilson loop. Each term of the series in $\Theta$ has associated various linking numbers, such that, at the $n$th term of the expansion, there will arise $6^{n}$ extra linking numbers. All these extra terms correspond to new homology cycles generated by the nonvanishing parameter $\Theta$.

The linking number is ordinarily a topological invariant; now the noncommutative linking numbers considered here will represent a topological invariant of the corresponding more general noncommutative topology. Thus, the arising extra terms and their involved mathematical structures deserve a detailed mathematical and physical interpretation and further analysis. In the present paper we will restrict ourselves to compute the first order noncommutative corrections to the linking numbers. This should be considered a first step of a description of the subject.

To explore how the modifications to topology of knots are immersed on the noncommutative space, we consider the abelian Jones-Witten polynomials in the path integral formalism which are given in terms of Wilson loops. We will show explicitly that the polynomials are changed due to noncommutativity already at the first order; there will be a nonvanishing and nontrivial modification of the linking numbers due the noncommutative generalization of the notion of topology.

In order to explore our proposal in a more detailed way we consider the application of the noncommutative Wilson loops to the Aharonov-Bohm effect. Some literature on the noncommutative Aharonov-Bohm effect and its relation to the Landau levels can be found in [42-47].

It is worth mentioning that the Wilson loops have been used in the description of some quantum theories of gravity. Some of these results can be found in [48-51]. The results found in the present paper would be applied also for this kind of theories.

This paper is structured as follows. In Section 2 we overview the Seiberg-Witten map and set the notation and conventions that we will follow along the paper. Section 3 is devoted to constructing the noncommutative Wilson loops based on the gauge field of the Seiberg-Witten map. In Section 4 we introduce the linking numbers; first in order to explore the geometrical properties of noncommutative Wilson loops we use basic ideas of Poincaré duality and interpret higher-order powers in the Seiberg-Witten expansion in terms of the arising new linking numbers. In Section 5 we compute the abelian Jones-like polynomials using the path integral formalism through the Chern-Simons functional up to first order in the noncommutative parameter. As a physical application, in Section 6 we explore the abelian noncommutative Aharonov-Bohm effect by means of the gauge field of the Seiberg-Witten map. The noncommutative Aharonov-Bohm effect is a very interesting physical example in which the noncommutativity could be relevant. It has known effects already described in the literature [42-47]. Moreover it has a relation with the noncommutative Landau levels. We will find that our results are consistent with these mentioned results. Section 7 is devoted to final remarks.

\section{The Seiberg-Witten Map}

Our aim is not to provide an extensive review on the SeibergWitten map [3] but to only recall the relevant structure which will be needed in the following sections. Throughout this paper we will follow the notation and conventions introduced in [7].

We are interested in noncommutativity utilizing the Seiberg-Witten map [3]. This proposal was extended in [57] for any gauge field coupled to matter. In the following we present a brief description of this construction.

The central idea is to deform the algebraic structure of continuous spaces in particular the polynomials in $N$ variables generated by powers of $x^{I}$ where $I=0, \ldots, N$, which 
is a freely generated algebra $\mathbb{C}\left[x^{1}, \ldots, x^{N}\right]$. Now consider the usual commutations relations between the coordinates

$$
\left[x^{\mu}, x^{\nu}\right]=0 .
$$

This algebraic structure will be deformed assuming

$$
\left[\widehat{x}^{\mu}, \widehat{x}^{\nu}\right]=i \Theta^{\mu \nu},
$$

where $\Theta^{\mu \nu}=-\Theta^{\nu \mu} \in \mathbb{R}$; that is, $\Theta^{\mu \nu}$ is the noncommutative parameter and $\hat{x}$ are the noncommutative coordinates (as we can see this algebra is similar to the Heisenberg algebra in the phase space); for a formal description see [7].

Explicitly this modifies the way we multiply polynomials and in general functions over the noncommutative variables in terms of the commutative variables through the Moyal $\star$ product defined by

$$
f \star g(x)=\mu\left(e^{(i / 2) \Theta^{\rho \sigma} \partial_{\rho} \otimes \partial_{\sigma}} f \otimes g\right),
$$

where $\mu$ is the product map defined by $\mu(f(x) \otimes g(x))=f(x)$. $g(x)$.

In this context the gauge transformations of a matter field $\Psi(x)$ are defined as

$$
\delta_{\Lambda} \Psi(x)=i \Lambda \star \Psi(x)
$$

where $\Lambda(x)$ is the noncommutative gauge parameter which is Lie algebra-valued; that is, $\Lambda(x)=\Lambda^{a}(x) T^{a}$. Now let us compute explicitly the following variation of the field $\Psi$ :

$$
\begin{aligned}
& \left(\delta_{\Lambda_{1}} \delta_{\Lambda_{2}}-\delta_{\Lambda_{1}} \delta_{\Lambda_{2}}\right) \Psi=\left(\Lambda_{1} \star \Lambda_{2}-\Lambda_{2} \star \Lambda_{1}\right) \star \Psi \\
& =\frac{1}{2}\left(\left[\Lambda_{1}^{a} \stackrel{\star}{,} \Lambda_{2}^{b}\right]\left\{T^{a}, T^{b}\right\}+\left\{\Lambda_{1}^{a} \stackrel{\star}{,} \Lambda_{2}^{b}\right\}\left[T^{a}, T^{b}\right]\right) \\
& \quad \star \Psi .
\end{aligned}
$$

It is worth mentioning that the fields are not Lie algebravalued because not only do we have commutators but also we have anticommutators. So the algebra that posses both operations (commutators and anticommutators) is precisely the Universal Enveloping Algebra.

The covariant derivative is defined by

$$
D_{\mu}^{\star} \Psi(x)=\partial_{\mu} \Psi(x)-i \widehat{A}_{\mu} \star \Psi(x),
$$

where $\widehat{A}_{\mu}$ is the noncommutative gauge field and transforms as

$$
\delta_{\Lambda} \widehat{A}_{\mu}=\partial_{\mu} \Lambda+i\left[\Lambda * \widehat{A}_{\mu}\right] .
$$

We can see that these terms have infinity many degrees of freedom, but in [3] it was shown that all the higherorder terms depend only on the zeroth order terms (the commutative term), that is, of the gauge parameter $\Lambda^{(0) a} T^{a}$ and the gauge field $A_{\mu}^{(0) a} T^{a}$. Let us assume that the gauge parameter $\Lambda_{\alpha}$ depends only on $\alpha$ and $A_{\mu}$, that is, the gauge parameter and the gauge field, respectively. With these assumptions borne in mind, let us substitute in expression (5); then we have

$$
\Lambda_{\alpha} \star \Lambda_{\beta}-\Lambda_{\beta} \star \Lambda_{\alpha}+i\left(\delta_{\alpha} \Lambda_{\beta}-\delta_{\beta} \Lambda_{\alpha}\right)=i \Lambda_{-i[\alpha, \beta]} .
$$

This expression could be solved perturbatively assuming an expansion in the parameter $\Theta$ as $\Lambda_{\alpha}=\Lambda_{\alpha}^{(0)}+\Lambda_{\alpha}^{(1)}+\cdots$, where $\Lambda_{\alpha}^{(0)}=\alpha=\alpha^{a} T^{a}$.

For example, up to first order in $\Theta$, we find the following expression:

$$
\begin{aligned}
& \delta_{\alpha} \Lambda_{\beta}^{(1)}-\delta_{\beta} \Lambda_{\alpha}^{(1)}-i\left[\alpha, \Lambda_{\beta}^{(1)}\right]-i\left[\Lambda_{\alpha}^{(1)}, \beta\right]-\Lambda_{-i[\alpha, \beta]}^{(1)} \\
& =-\frac{1}{2} \Theta^{\mu \nu}\left\{\partial_{\mu} \alpha, \partial_{\gamma} \beta\right\},
\end{aligned}
$$

whose solution is $\Lambda_{\alpha}^{(1)}=\alpha-(1 / 4) \Theta^{\mu \nu}\left\{A_{\mu}, \partial_{\gamma} \alpha\right\}$. With this expression we can compute in a similar way the expression for matter fields assuming the transformation at 0 -th order $\delta_{\alpha} \Psi^{(0)}=i \alpha \Psi^{(0)}$.

For the gauge field we expand again in orders of $\Theta$ as $\widehat{A}=$ $A^{(0)}+A^{(1)}+A^{(2)}+\cdots$ and substituting it into (7), up to first order in $\Theta$, we obtain

$$
A_{\mu}^{(1)}=\frac{1}{4} \Theta^{\rho \sigma}\left(\left\{F_{\rho \mu}, A_{\sigma}\right\}-\left\{A_{\rho}, \partial_{\sigma} A_{\mu}\right\}\right) .
$$

Finally the field strength tensor is given by $F_{\mu \nu}^{\star}=i\left[D_{\mu}^{\star}, d_{\nu}^{\star}\right]$, whose solution up to first order is

$$
\begin{aligned}
\widehat{F}_{\mu \nu} & =F_{\mu \nu}+\frac{1}{4} \\
\cdot & \Theta^{\rho \sigma}\left(2\left\{F_{\rho \mu}, F_{\sigma \nu}\right\}+\left\{D_{\rho} F_{\mu \nu}, A_{\sigma}\right\}-\left\{A_{\rho}, \partial_{\sigma} F_{\mu \nu}\right\}\right) .
\end{aligned}
$$

Here we can identify $F_{\mu \nu}^{(0)}=F_{\mu \nu}$ and $F_{\mu \nu}^{(1)}=$ $(1 / 4) \Theta^{\rho \sigma}\left(2\left\{F_{\rho \mu}, F_{\sigma \nu}\right\}+\left\{D_{\rho} F_{\mu \nu}, A_{\sigma}\right\}-\left\{A_{\rho}, \partial_{\sigma} F_{\mu \nu}\right\}\right)$.

\section{Noncommutative Wilson Loops}

The usual Wilson loop is given by the following expression:

$$
W(C)=\operatorname{Tr} P \exp \left(\frac{i}{\hbar} \int_{C} A\right)
$$

where $A=A_{\mu}^{a} t^{a} d x^{\mu}$, with $t^{a}$ being the Lie algebra generators; then the Wilson loop is an element of the Lie group and an element of the holonomy. An extension to the noncommutative case was proposed in a straightforward manner using the Moyal product in $[30,32]$. In this work we make use of the gauge field present in the Seiberg-Witten map in order to construct the corresponding noncommutative Wilson loop. This leads to change in the connection $A$ by $\widehat{A}$ which possesses an expansion in powers in $\Theta$ given by $\widehat{A}=A^{(0)}+A^{(1)}+A^{(2)}+\cdots$ and every $A^{(i)}$ is expanded in terms of the usual 1-form basis; that is, $A^{(i)}=A_{\mu}^{(i)} d x^{\mu}$; the Wilson loop corresponding to the Seiberg-Witten map is given by

$$
\widehat{W}(C)=\operatorname{Tr} P \exp { }_{\star}\left(\frac{i}{\hbar} \int_{C} \widehat{A}\right) .
$$


For Chern-Simons (our case of interest) it was shown in [28] that the same formulae apply for the Seiberg-Witten map. Further developments for higher-order computations of the Seiberg-Witten map can be found in [52].

The $\star$-exponential is defined as

$$
\begin{aligned}
& \operatorname{Tr} \exp _{\star}\left(\frac{i}{\hbar} \int_{C} \widehat{A}\right) \\
& =1+\frac{i}{\hbar} \int_{C} \operatorname{Tr} \widehat{A}(x) \\
& +\frac{i^{2}}{\hbar^{2} 2 !} \int_{C} \int_{C} \operatorname{Tr}(\widehat{A}(x) \star \widehat{A}(y)) \\
& +\frac{i^{3}}{\hbar^{3} 3 !} \int_{C} \int_{C} \int_{C} \operatorname{Tr}(\widehat{A}(x) \star \widehat{A}(y) \star \widehat{A}(z)) \\
& +\cdots,
\end{aligned}
$$

where we are assuming the integration order $x<y<z<\cdots$, with $x, y, z, \ldots \in \mathbb{C}$.

3.1. Abelian Case. For the abelian case the Wilson loop can be written as follows:

$$
\begin{gathered}
\widehat{W}(C)=\exp _{\star}\left(\frac{i}{\hbar} \int_{C} \widehat{A}\right)=\exp _{\star}\left(\frac{i}{\hbar} \int_{C} A^{(0)}\right) \\
\cdot \exp _{\star}\left(\frac{i}{\hbar} \int_{C} A^{(1)}\right) \exp _{\star}\left(\frac{i}{\hbar} \int_{C} A^{(2)}\right) \cdots
\end{gathered}
$$

Then we can compute explicitly the Wilson loop up to second $\Theta$ order. We can see that it is necessary to expand the first three exponentials:

$$
\begin{aligned}
& \widehat{W}(C) \approx \exp _{\star}\left(\frac{i}{\hbar} \int_{C} A^{(0)}\right) \\
& \cdot \exp _{\star}\left(\frac{i}{\hbar} \int_{C} A^{(1)}\right) \exp _{\star}\left(\frac{i}{\hbar} \int_{C} A^{(2)}\right) .
\end{aligned}
$$

It is easy to check that the exponential $\exp _{\star}\left((i / \hbar) \int_{C} A^{(j)}\right)$ up to order $2 j+1$ is given by

$$
\begin{aligned}
& \exp _{\star}\left(\frac{i}{\hbar} \int_{C} A^{(j)}\right)=1+\frac{i}{\hbar} \int_{C} A^{(j)}+\frac{i^{2}}{2 ! \hbar^{2}} \\
& \cdot \int_{C \times C} A^{(j)} \star A^{(j)}+\frac{i^{3}}{3 ! \hbar^{3}} \int_{C \times C \times C} A^{(j)} \star A^{(j)} \star A^{(j)} \\
& +\cdots \approx \exp \left(\frac{i}{\hbar} \int_{C} A^{(j)}\right) \\
& \cdot\left[1+\frac{i^{2}}{2 ! \hbar^{2}} \frac{i}{2} \Theta^{\mu \nu} \partial_{\mu} \int_{C} A^{(j)} \cdot \partial_{\nu} \int_{C} A^{(j)}\right]
\end{aligned}
$$

The second term vanishes in virtue of the abelianity and the skew symmetry of $\Theta^{\mu \nu}$; thus the second order Wilson loop is given by

$$
\begin{gathered}
\widehat{W}(C) \approx \exp \left(\frac{i}{\hbar} \int_{C} A^{(0)}\right)\left[1+\frac{i}{\hbar} \int_{C} A^{(1)}+\frac{i}{\hbar} \int_{C} A^{(2)}\right. \\
\left.+\frac{1}{2 !}\left(\frac{i}{\hbar}\right)^{2} \int_{C} A^{(1)} \int_{C} A^{(1)}\right]=\exp \left(\frac{i}{\hbar} \alpha_{0}\right)[1 \\
\left.+\frac{i}{\hbar} \Theta \alpha_{1}+\frac{i}{\hbar} \Theta^{2} \alpha_{2}+\frac{1}{2 !}\left(\frac{i}{\hbar}\right)^{2} \Theta^{2} \alpha_{1} \alpha_{1}\right] .
\end{gathered}
$$

As an example let us compute $\widehat{W}(C)$ assuming a pure constant magnetic field along the $z$-axis $\vec{B}=B_{0} \widehat{k}$ and $C=S^{1}$ in the $x-y$ plane; hence $A_{1}^{0}=-\left(B_{0} / 2\right) y$ and $A_{2}^{0}=\left(B_{0} / 2\right) x$; thus

$$
\begin{aligned}
\alpha_{0} & =\int_{C} A_{\mu}^{(0)} d x^{\mu}=\frac{B_{0}}{2}(2 \pi)=\phi \\
\Theta \alpha_{1} & =\int_{C} A_{\mu}^{(1)} d x^{\mu}=\frac{3}{4} B_{0}^{2}(2 \pi) \Theta^{12}=\frac{3}{2 \pi} \phi^{2} \Theta^{12}, \\
\Theta^{2} \alpha_{2} & =\int_{C} A_{\mu}^{(2)} d x^{\mu}=\frac{1}{2} B_{0}^{3}(2 \pi)\left(\Theta^{12}\right)^{2} \\
& =\frac{4}{(2 \pi)^{2}} \phi^{3}\left(\Theta^{12}\right)^{2},
\end{aligned}
$$

where $\phi$ is the flux due to the commutative field through the closed curve $C$. Now up to second order the noncommutative Wilson line is given by

$$
\begin{aligned}
& \widehat{W}(C) \approx \exp \left(\frac{i}{\hbar} \alpha_{0}\right)\left(1+\Theta \frac{i}{\hbar} \alpha_{1}\right. \\
& \left.+\Theta^{2}\left[\frac{i}{\hbar} \alpha_{2}+\frac{1}{2}\left(\frac{i}{\hbar}\right)^{2} \alpha_{1}^{2}\right]\right)=\exp \left(\frac{i}{\hbar} \frac{B_{0}}{2}(2 \pi)\right)(1 \\
& +\frac{i}{\hbar} \Theta^{12}\left[\frac{3}{4} B_{0}(2 \pi)\right] \\
& \left.+\frac{1}{2} \frac{i}{\hbar}\left(\Theta^{12}\right)^{2}\left[(2 \pi) B_{0}^{3}+\frac{i}{\hbar}\left(\frac{3}{4} B_{0}^{2}\right)^{2}(2 \pi)^{2}\right]\right)
\end{aligned}
$$

Therefore, as we might expect, to observe noncommutativity effects assuming $\Theta \ll 1$ we need the intensity of the magnetic field $B_{0}$ to be large enough.

\section{Linking Numbers and the Wilson Loops}

It is well known that on a three-manifold $M=S^{3}$ or $\mathbb{R}^{3}$ the magnetic field induced by a loop wire carrying a current is proportional to the linking number between the loop and the magnetic lines, due to the Biot-Savart law (which is essentially the Gauss linking number). This is written usually as

$$
\Phi=\int_{\Sigma} F^{(0)}=\int_{\Sigma} d A^{(0)}=\int_{C} A^{(0)},
$$

where we are assuming that $C$ is a trivial homology 1-cycle (i.e., a trivial element in the first homology group). Thus there 
exists a 2 -chain $\Sigma$ (it could be regarded as a two-dimensional submanifold of $\mathbb{R}^{3}$ ) such that $C=\partial \Sigma$ is immersed on the three-dimensional Euclidean space. The connection $A^{(0)}=$ $A_{\mu}^{(0)} d x^{\mu}$ is the commutative (usual) magnetic potential 1form. As we can see the last integral is the phase that appears in the commutative Wilson loop.

Since $C$ is a trivial cycle by Poincaré duality there exists $d \eta$ where $\eta$ is a 1 -form. The mathematical interpretation assuming that $C$ is a trivial 1 -cycle and the flux is nonzero implies that $A^{(0)}$ is not a closed form; that is,

$$
\Phi=\int_{M} A^{(0)} \wedge d \eta=\int_{M} \eta \wedge d A^{(0)}
$$

In the previous integral we integrate out by parts and in this way we can regard $d A^{(0)}$ as the Poincaré dual of some trivial cycle $C^{(0)}$ which is boundary of the 2 -chain $\Sigma^{(0)}$. Hence this integral represents the linking number among the trivial cycles $C$ and $C^{(0)}$ where the respective boundaries of the 2 cycles are $\Sigma$ and $\Sigma^{(0)}$.

Now we will consider the noncommutative case; then the phase in the noncommutative Wilson loop assuming the expansion on $\Theta$ is given by

$$
\int_{C} \widehat{A}=\int_{C} A^{(0)}+\int_{C} A^{(1)}+\int_{C} A^{(2)}+\cdots .
$$

Interpreting this in terms of linking numbers, the first term is the link number between $C$ and $C^{(0)}$ and the second term is interpreted in the following way. Let us consider from the Seiberg-Witten map the first order potential $A_{\mu}^{(1)}=$ $-\Theta^{\kappa \lambda}(1 / 2) A_{\kappa}^{(0)}\left(\partial_{\lambda} A_{\mu}^{(0)}+F_{\lambda \mu}^{(0)}\right)$ that can be arranged as

$$
A^{(1)}=\Theta^{\kappa \lambda} A_{\kappa \lambda}^{(1)}=\Theta^{\kappa \lambda} A_{\kappa \lambda \mu}^{(1)} d x^{\mu} .
$$

In this way $A^{(1)}$ can be regarded as the sum of six 1-forms $A_{\kappa \lambda}^{(1)}=A_{\kappa \lambda \mu}^{(1)} d x^{\mu}$ (since we are working in $\mathbb{R}^{3}$ )

$$
\int_{C} A^{(1)}=\int_{M} A^{(1)} \wedge d \eta=\int_{M} \eta \wedge d A^{(1)}=\int_{C^{(1)}} \eta,
$$

where $C^{(1)}$ is a trivial 1-cycle. But explicitly the first integral $\Theta^{\kappa \lambda} \int_{C} A_{\kappa \lambda}^{(1)}$ must be equal to $\int_{C^{(1)}} \eta$; therefore, it also will be expanded in $\Theta$; then (25) reads

$$
\Theta^{\kappa \lambda} \int_{C} A_{\kappa \lambda}^{(1)}=\Theta^{\kappa \lambda} \int_{C_{\kappa \lambda}^{(1)}} \eta
$$

This equation implies that $C_{\kappa \lambda}^{(1)}=\partial \Sigma_{\kappa \lambda}^{(1)}$ is a trivial cycle; thus it can be regarded as the linking number between $C$ and each $C_{\kappa \lambda}^{(1)}$; for example, in the three-dimensional Euclidean space

$$
\begin{aligned}
\Theta^{\kappa \lambda} \int_{C_{\kappa \lambda}^{(1)}} \eta= & \Theta^{12}\left(\int_{C_{12}^{(1)}} \eta-\int_{C_{21}^{(1)}} \eta\right) \\
& +\Theta^{13}\left(\int_{C_{13}^{(1)}} \eta-\int_{C_{31}^{(1)}} \eta\right) \\
& +\Theta^{23}\left(\int_{C_{23}^{(1)}} \eta-\int_{C_{32}^{(1)}} \eta\right),
\end{aligned}
$$

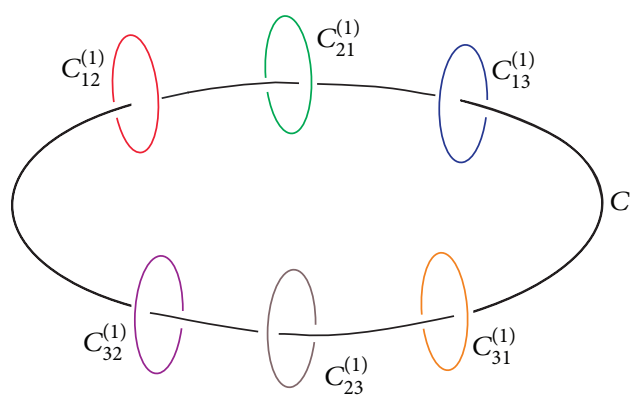

FIGURE 1: The figure accounts the computation at the first order in $\Theta$ of the linking number. For the case of knots in $\mathbb{R}^{3}$ it is observed at this order that the noncommutativity induces the existence of 6 different knots, represented in the simplest case by circles of different colors $C_{\kappa \lambda}^{(1)}$, that intersect the original trivial homology 1-cycle C. At the $n$th order there will be $6^{n}$ knots intersecting $C$. In the general case the knots $C_{\kappa \lambda}^{(1)}$ would be real knots linked, for instance, the trefoil or even more complicated knots.

or

$$
\begin{aligned}
\Theta^{\kappa \lambda} \int_{C} A_{\kappa \lambda}^{(1)}= & \Theta^{12} \int_{C}\left(A_{12}^{(1)}-A_{21}^{(1)}\right) \\
& +\Theta^{13} \int_{C}\left(A_{13}^{(1)}-A_{31}^{(1)}\right) \\
& +\Theta^{23} \int_{C}\left(A_{23}^{(1)}-A_{32}^{(1)}\right)
\end{aligned}
$$

in general since $A_{\kappa \lambda}^{(1)} \neq A_{\lambda \kappa}^{(1)}$, then the linking number between $C$ and $C_{\kappa \lambda}^{(1)}$ is different from the one of $C$ and $C_{\lambda_{\kappa}}^{(1)}$. In Figure 1 the intersection between the $C_{\lambda_{\kappa}}^{(1)} 1$-cycles is explicitly displayed, represented in different colors by the simplest case when they are circles and the original 1-cycle $C$.

Let us introduce the following notation: $\operatorname{Lk}\left(C_{1}, C_{2}\right)$ denotes the linking number between the homologically trivial 1-cycles $C_{1}$ and $C_{2}$.

Now consider the second order term and reordering the expression $A^{(2)}$ in the Seiberg-Witten map, it could be written in a similar way as in (24):

$$
A^{(2)}=\Theta^{\kappa_{1} \lambda_{1}} \Theta^{\kappa_{2} \lambda_{2}} A_{\kappa_{1} \lambda_{1} \kappa_{2} \lambda_{2} \mu}^{(2)} d x^{\mu} .
$$

Let $A_{\kappa_{1} \lambda_{1} \kappa_{2} \lambda_{2}}^{(2)}=A_{\kappa_{1} \lambda_{1} \kappa_{2} \lambda_{2} \mu}^{(2)} d x^{\mu}$, in analogy to the first order
term

$$
\Theta^{\kappa_{1} \lambda_{1}} \Theta^{\kappa_{2} \lambda_{2}} \int_{C} A_{\kappa_{1} \lambda_{1} \kappa_{2} \lambda_{2}}^{(2)}=\Theta^{\kappa_{1} \lambda_{1}} \Theta^{\kappa_{2} \lambda_{2}} \int_{C_{\kappa_{1} \lambda_{1} \kappa_{2} \lambda_{2}}^{(2)}} \eta ;
$$

again here $d \eta$ stands for the Poincaré dual of $\gamma$. As we can see in general the total flux induced by the second order term is the sum of the 36 linking numbers $\operatorname{Lk}\left(C, C_{\kappa_{1} \lambda_{1} \kappa_{2} \lambda_{2}}^{(2)}\right)$.

Finally in general for the $m$ th order we rearrange the expression $A^{(m)}$ from the Seiberg-Witten map; we rewrite it as

$$
A^{(m)}=\Theta^{\kappa_{1} \lambda_{1}} \cdots \Theta^{\kappa_{m} \lambda_{m}} A_{\kappa_{1} \lambda_{1} \cdots \kappa_{m} \lambda_{m} \mu}^{(m)} d x^{\mu}
$$


We define $A_{\kappa_{1} \lambda_{1} \cdots \kappa_{m} \lambda_{m}}^{(m)}=A_{\kappa_{1} \lambda_{1} \cdots \kappa_{m} \lambda_{m} \mu}^{(m)} d x^{\mu}$. Thus we have

$$
\begin{aligned}
& \Theta^{\kappa_{1} \lambda_{1}} \cdots \Theta^{\kappa_{m} \lambda_{m}} \int_{C} A_{\kappa_{1} \lambda_{1} \cdots \kappa_{m} \lambda_{m}}^{(m)} \\
& =\Theta^{\kappa_{1} \lambda_{1}} \cdots \Theta^{\kappa_{m} \lambda_{m}} \int_{C_{\kappa_{1} \lambda_{1} \cdots k_{m} \lambda_{m}}^{(m)}} \eta \\
& =\Theta^{\kappa_{1} \lambda_{1}} \cdots \Theta^{\kappa_{m} \lambda_{m}} \operatorname{Lk}\left(C, C_{\kappa_{1} \lambda_{1} \cdots \kappa_{m} \lambda_{m}}^{(m)}\right),
\end{aligned}
$$

where $C_{\kappa_{1} \lambda_{1} \cdots \kappa_{m} \lambda_{m}}^{(m)}$ are homologically trivial 1-cycles; that is, a 2-chain $\sum_{\kappa_{1} \lambda_{1} \cdots \kappa_{m} \lambda_{m}}^{(m)}$ exists, such that $C_{\kappa_{1} \lambda_{1} \cdots \kappa_{m} \lambda_{m}}^{(m)}=$ $\partial \sum_{\kappa_{1} \lambda_{1} \cdots \kappa_{m} \lambda_{m}}^{(m)}$. Therefore, in general the $m$-flux through $C$ could be regarded as the sum of $6^{m}$ linking numbers between $C$ and $C_{\kappa_{1} \lambda_{1} \cdots \kappa_{m} \lambda_{m}}^{(m)}$ in $\mathbb{R}^{3}$.

\section{The Jones-Witten-Like Invariants}

The Jones polynomials in Witten's path integral formulation are given by the correlation functions of Wilson loops

$$
\begin{aligned}
J_{C} & =\langle W(C)\rangle=\frac{1}{N} \int \mathscr{D} A \\
& \cdot \exp \left[\frac{i}{\hbar} k \int_{M} \operatorname{Tr}(A \wedge d A+A \wedge A \wedge A)\right] \\
& \cdot \operatorname{Tr}_{R} W(C),
\end{aligned}
$$

where $W(C)=P \exp \left[(i / \hbar) \int_{C} A\right]$ is the Wilson line, $R$ is a representation of the gauge group, $A$ is the gauge field, $C$ is a knot (homologically trivial 1-cycle), $M$ is the Euclidean 3 -dimensional space or the unitary 3-sphere, and $N$ is the normalization factor.

We will consider the simplest case when the gauge group is $U(1)$ but consider the noncommutative Wilson loop (abelian case) and compute the first nontrivial correction which will arise at first order in $\Theta$.

First of all we change in the path integral $A$ by $\widehat{A}$; then, the noncommutative Jones-Witten-like invariants which will depend explicitly on the noncommutative parameter (for the abelian case) read

$$
\begin{aligned}
& J_{C}(\Theta)=\langle\widehat{W}(C)\rangle=\frac{1}{N} \int \mathscr{D} \widehat{A} \\
& \cdot \exp \left[\frac{i}{\hbar} k \int_{M} \operatorname{Tr}\left(\widehat{A} \wedge^{\star} d \widehat{A}+\widehat{A} \wedge^{\star} \widehat{A} \wedge^{\star} \widehat{A}\right)\right] \\
& \cdot \operatorname{Tr}_{R} \widehat{W}(C) .
\end{aligned}
$$

Since we will consider the $\Theta$-expansion just up to first order, the term $\widehat{A} \stackrel{\star}{\wedge} \widehat{A} \wedge \widehat{A}$ does not contribute (it contributes up to third order); then we get the following expression for the action:

$$
\begin{aligned}
\exp & {\left[\frac{i}{\hbar} k \int_{M}\left(A^{(0)} \wedge d A^{(0)}+2 A^{(0)} \wedge d A^{(1)}\right)\right] } \\
\approx \exp & {\left[\frac{i}{\hbar} k \int_{M} A^{(0)} \wedge d A^{(0)}\right] } \\
\cdot & {\left[1+\frac{i}{\hbar} 2 k \Theta^{\kappa \lambda} \int_{M} A^{(0)} \wedge d A_{\kappa \lambda}^{(1)}\right], }
\end{aligned}
$$

and the noncommutative Wilson line (13) up to first order in $\Theta$ is

$$
\begin{aligned}
\widehat{W}(C) & =\exp _{\star}\left(\frac{1}{\hbar} \int_{C} \widehat{A}\right) \\
& \approx \exp \left(\frac{i}{\hbar} \int_{C} A^{(0)}\right)\left[1+\frac{i}{\hbar} \Theta^{\kappa \lambda} \int_{C} A_{\kappa \lambda}^{(1)}\right] .
\end{aligned}
$$

Then the functional integral up to first order (the first $\Theta$ order Jones polynomials) is given by

$$
\begin{gathered}
\int \mathscr{D} A^{(0)} \exp \left(\frac{i}{\hbar} k \int_{M} A^{(0)} \wedge d A^{(0)}\right) \exp \left(\frac{i}{\hbar} \int_{C} A^{(0)}\right) \\
\cdot\left[1+\frac{i}{\hbar} \Theta^{\kappa \lambda} \int_{C} A_{\kappa \lambda}^{(1)}+\frac{i}{\hbar} 2 k \Theta^{\kappa \lambda} \int_{M} A^{(0)} \wedge d A_{\kappa \lambda}^{(1)}\right] .
\end{gathered}
$$

The integral is performed just on the commutative term $A^{(0)}$ since every order can be written in term $A^{(0)}$ in virtue of Seiberg-Witten map. Rewriting the previous expression we get

$$
\begin{aligned}
& \int \mathscr{D} A^{(0)} \exp \left(\frac{i}{\hbar} k \int_{M}\left(A^{(0)} \wedge d A^{(0)}+\frac{1}{k} A^{(0)} \wedge d \eta\right)\right) \\
& \cdot\left[1+\frac{i}{\hbar} \Theta^{\kappa \lambda} \int_{M} A_{\kappa \lambda}^{(1)} \wedge d \eta+\frac{i}{\hbar} 2 k \Theta^{\kappa \lambda} \int_{M} A^{(0)}\right. \\
& \left.\wedge d A_{\kappa \lambda}^{(1)}\right]
\end{aligned}
$$

where $d \eta$ is the Poincaré dual of the knot $C$. Next we need to compute these three integrals; the first one is the usual abelian Jones polynomial-like for the knot $C$, whose evaluation is proportional to

$$
\exp \left(-\frac{i}{4 \hbar k} \int_{M} \eta \wedge d \eta\right)
$$

where $\int_{M} \eta \wedge d \eta$ is the Hopf invariant or self-linking number. Now we consider the second integral which is rewritten as

$$
\begin{aligned}
& \frac{i}{\hbar} \Theta^{\kappa \lambda} \int \mathscr{D} A^{(0)} \\
& \cdot \exp \left[\frac{i}{\hbar} k \int_{M}\left(A^{(0)} \wedge d A^{(0)}+\frac{1}{k} A^{(0)} \wedge d \eta\right)\right] \\
& \quad \cdot\left[\int_{M} A_{\kappa \lambda}^{(1)} \wedge d \eta\right] .
\end{aligned}
$$


First of all, notice that any 1 -form $A^{(0)}$, using the Hodge decomposition theorem, can be uniquely decomposed as $A^{\text {harm }}+d B+\delta C$, where $A^{\text {harm }}$ is a harmonic 1-form (i.e., it is a solution to the laplacian $\left.\Delta_{p}=d \delta+\delta d\right), B$ is a 0 -form, and $C$ is a 2 -form. Here $d$ is the usual exterior derivative (which maps a $p$-form into $(p+1)$-form) and $\delta=(-1)^{n(p+1)+1} * d *$ is its adjoint operator (which maps $p$-form into $(p-1)$-form). The $*$ stands for the Hodge star operator which maps $p$ form into $(n-p)$-form. Also it is necessary to consider that $*^{2}=(-1)^{p(n-p)}$.

In order to integrate out the path integral measure is

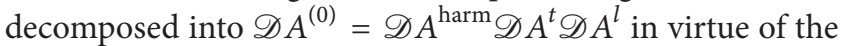
Hodge decomposition theorem, where $A^{l}=d B$ and $A^{t}=\delta C$ are the longitudinal and transversal parts of $A^{(0)}$, respectively. It is well known that just the transversal parts contribute to the integration.

Moreover, it is necessary to introduce some extra conventions. Let $\Lambda_{p}$ be the space of eigenforms of $\Delta_{p}$ with nonvanishing eigenvalue $\lambda^{2}$. This space can be decomposed into $\Lambda_{p}^{l}$ and $\Lambda_{p}^{t}$, that is, into the longitudinal and transversal parts. We also need to consider the following maps: $d$ : $\Lambda_{p}^{t} \rightarrow \Lambda_{p+1}^{l}, \delta: \Lambda_{p+1}^{l} \rightarrow \Lambda_{p}^{t}$, and an isomorphism given by $*: \Lambda_{p} \rightarrow \Lambda_{n-p}$ among forms with the same eigenvalue $\lambda^{2}$. Given these maps finally we construct the following isomorphism:

$$
\lambda^{-1} * d: \Lambda_{p}^{t} \longrightarrow \Lambda_{n-p-1}^{t}
$$

Since we will consider $M=S^{3}$ or $\mathbb{R}^{3}$ and 1 -forms (this is $n=3$ and $p=1$ ), the last isomorphism is reduced to $\lambda^{-1} * d$ : $\Lambda_{1}^{t} \rightarrow \Lambda_{1}^{t}$.

Now consider a basis of transverse 1-eigenforms $\left\{A_{i}^{t}\right\}$ such that it satisfies the normalization condition $\left\langle A_{i}^{t} \mid A_{j}^{t}\right\rangle=$ $\int_{M} A_{i}^{t} \wedge * A_{j}^{t}=\delta_{i j}$ (inner product among 1 -forms). We expand the transversal part of $A^{(0)}$ and $\eta$ in terms of this basis; hence $A^{t}=\sum_{i} a_{i}^{(0)} A_{i}^{t}$ and $\eta^{t}=\sum_{i} \eta_{i} A_{i}^{t}$. With the aid of the inner product, $*^{2}=+1$, for 2 -forms in a Riemannian manifold and the isomorphism (41), the term in the exponential on the left-hand side of (40) can be rewritten as follows:

$$
\begin{gathered}
k\left\langle A^{(0)} \mid * d A^{(0)}\right\rangle+\left\langle A^{(0)} \mid * d \eta\right\rangle \\
=k \sum_{i} \lambda_{i}\left(\left(a_{i}^{(0)}\right)^{2}+\frac{1}{k} a_{i}^{(0)} \eta_{i}\right)
\end{gathered}
$$

where $* d A^{(0)}=* d \sum_{i} a_{i}^{(0)} A_{i}^{t}=\sum_{i} a_{i}^{(0)} * d A_{i}^{t}=\sum_{i} a_{i}^{(0)} \lambda_{i} A_{i}^{t}$ and similarly for $* d \eta$.

In a similar spirit we compute the first order $\Theta$ expansion of the Wilson line

$$
\Theta^{\kappa \lambda} \int_{M} A_{\kappa \lambda}^{(1)} \wedge d \eta=\int_{M} A^{(1)} \wedge d \eta=\sum_{i} \lambda_{i} a_{i}^{(1)} \eta_{i}
$$

where $A^{(1)}=\sum a_{\ell}^{(1)} A_{\ell}^{t}$. Explicitly $a_{\ell}^{(1)}$ can be written in terms of $a^{(0)}$ since $A_{\mu}^{(1)}=-(1 / 2) \Theta^{\kappa \lambda} A_{\kappa}^{(0)}\left(\partial_{\lambda} A_{\mu}^{(0)}+F_{\lambda \mu}^{(0)}\right)$. Using the eigenbasis expansion $A^{(0)}=\sum_{i} a_{i}^{(0)} A_{i}^{t}$ and the relation

$$
d A_{i}^{t}=*^{2} d A_{i}^{t}=* \lambda_{i} A_{i}^{t}=\frac{1}{2} \lambda_{i} A_{i \mu}^{t} \varepsilon_{\rho \sigma}^{\mu} d x^{\rho} \wedge d x^{\sigma}
$$

where we use the fact that $*^{2}=1$. In virtue of the last expression we get the following useful expression for the partial derivatives of the gauge field components $\partial_{\rho} A_{i \sigma}=$ $(1 / 2) \lambda_{i} A_{i \mu}^{t} \varepsilon_{\rho \sigma}^{\mu}$; hence we can rewrite $A^{(1)}$ as

$$
A^{(1)}=\frac{3}{4} \Theta^{\kappa \lambda} \sum_{i, j} \lambda_{j} a_{i}^{(0)} a_{j}^{(0)} A_{i \kappa}^{t} A_{j \mu}^{t} \varepsilon_{\rho \lambda}^{\mu} d x^{\rho} .
$$

Finally projecting out $A^{(1)}$ into the transverse basis we get the following explicit expression:

$$
a_{\ell}^{(1)}=\int_{M} A^{(1)} \wedge * A_{\ell}=\frac{3}{8} \Theta^{\kappa \lambda} \sum_{i, j} \lambda_{j} a_{i}^{(0)} a_{j}^{(0)}\left[C_{i j \ell}\right]_{\kappa \lambda},
$$

where the C's are defined by the following expression:

$$
\left[C_{i j \ell}\right]_{\kappa \lambda}=\int_{M} \varepsilon^{\mu}{ }_{\gamma \lambda} \varepsilon^{\zeta}{ }_{\chi \rho} A_{i \kappa}^{t} A_{j \mu}^{t} A_{\ell \zeta}^{t} \cdot d x^{\gamma} \wedge d x^{\chi} \wedge d x^{\rho} .
$$

With these algebraic manipulations we can write (43) as

$$
\int_{M} A^{(1)} \wedge d \eta=\frac{3}{8} \Theta^{\kappa \lambda} \sum_{i, j, \ell} \eta_{\ell} \lambda_{j} \lambda_{\ell} a_{i}^{(0)} a_{j}^{(0)}\left[C_{i j \ell}\right]_{\kappa \lambda}
$$

Following a similar procedure we find

$$
\int_{M} A^{(0)} \wedge d A^{(1)}=\frac{3}{8} \Theta^{\kappa \lambda} \sum_{i, j, \ell} \lambda_{j} \lambda_{\ell} a_{i}^{(0)} a_{j}^{(0)} a_{\ell}^{(0)}\left[C_{i j \ell}\right]_{\kappa \lambda}
$$

Therefore, (40) can be reexpressed in terms of the previous expansion; then

$$
\begin{aligned}
& \frac{1}{N} \int \mathscr{D} A^{(0)} \exp \left[\frac{i}{\hbar}\right. \\
& \left.\cdot k \int_{M}\left(A^{(0)} \wedge d A^{(0)}+\frac{1}{k} A^{(0)} \wedge d \eta\right)\right] \cdot\left[\frac{i}{\hbar} \int_{M} A^{(1)}\right. \\
& \wedge d \eta]=\frac{1}{N} \int \prod_{m} d a_{m}^{(0)} \\
& \cdot \exp \left[\frac{i}{\hbar} k\left(\sum_{m} \lambda_{m}\left[\left(a_{m}^{(0)}\right)^{2}+\frac{1}{k} a_{m}^{(0)} \eta_{m}\right]\right)\right] \\
& \cdot\left[\frac{3}{8} \frac{i}{\hbar} \Theta^{\kappa \lambda} \sum_{i, j, \ell} \eta_{\ell} \lambda_{j} \lambda_{\ell} a_{i}^{(0)} a_{j}^{(0)}\left[C_{i j \ell}\right]_{\kappa \lambda}\right]
\end{aligned}
$$


where $N=\int \mathscr{D} A^{(0)} \exp \left[i k \int_{M} A^{(0)} \wedge d A^{(0)}\right]$ is a normalization factor which is given by

$$
N=\lim _{n \rightarrow \infty}\left(\frac{i \hbar \pi}{k}\right)^{n / 2} \frac{1}{\sqrt{\operatorname{det} \Delta}}
$$

where $\operatorname{det} \Delta=\prod_{m=1}^{n} \lambda_{m}$

In order to integrate out expression (50), it is convenient to rewrite it as follows:

$$
\begin{aligned}
& \frac{i}{\hbar} \Theta^{\kappa \lambda} \frac{3}{8} \sum_{\ell, m, i} \lambda_{\ell} \eta_{i} \lambda_{i}\left[C_{\ell m i}\right]_{\kappa \lambda} \int \prod_{n} d a_{n}^{(0)} \\
& \cdot \exp \left(-\frac{1}{2} \sum_{i, j} a_{i}^{(0)} A_{i j} a_{j}^{(0)}+\sum_{i} a_{i}^{(0)} J_{i}\right) a_{\ell}^{(0)} a_{m}^{(0)},
\end{aligned}
$$

where $A_{i j}=-(2 i / \hbar) k \lambda_{j} \delta_{i j}$ and $J_{i}=(i / \hbar) \lambda_{i} \eta_{i}$. Performing the integration we obtain

$$
\begin{aligned}
& \frac{1}{N} \int \mathscr{D} A^{(0)} \exp \left[\frac{i}{\hbar} k \int_{M}\left(A^{(0)} \wedge d A^{(0)}+\frac{1}{k} A^{(0)} \wedge d \eta\right)\right] \\
& \cdot\left[i \int_{M} A^{(1)} \wedge d \eta\right]=\frac{3}{8} \Theta^{\kappa \lambda} \sum_{i, j, \ell} \lambda_{j} \lambda_{\ell}\left[C_{i j \ell}\right]_{\kappa \lambda} \\
& \cdot \exp \left(-\frac{i}{4 k \hbar} \int_{M} \eta \wedge d \eta\right)\left(\frac{i}{4 \hbar k^{2}} \eta_{i} \eta_{j} \eta_{\ell}-\frac{1}{2 k}\right. \\
& \left.\quad \cdot \frac{\delta_{i j} \eta_{\ell}}{\lambda_{j}}\right) .
\end{aligned}
$$

Finally the third contribution in (38) associated with the factor $\int_{M} A^{(0)} \wedge d A^{(1)}$; then the term to compute is the following:

$$
\begin{aligned}
& \frac{2 i k}{\hbar N} \int \mathscr{D} A^{(0)} \exp \left[\frac{i}{\hbar}\right. \\
& \left.\cdot k \int_{M}\left(A^{(0)} \wedge d A^{(0)}+\frac{1}{k} A^{(0)} \wedge d \eta\right)\right]\left[\int_{M} A^{(0)}\right. \\
& \left.\wedge d A^{(1)}\right]=\frac{2 k}{N} \int \prod_{m} d a_{m}^{(0)} \\
& \cdot \exp \left[\frac{i}{\hbar} k\left(\sum_{m} \lambda_{m}\left[\left(a_{m}^{(0)}\right)^{2}+\frac{1}{k} a_{m}^{(0)} \eta_{m}\right]\right)\right] \\
& \cdot\left[\frac{3}{8} \frac{i}{\hbar} \Theta^{\kappa \lambda} \sum_{i, j, \ell} \lambda_{j} \lambda_{\ell} a_{i}^{0} a_{j}^{0} a_{\ell}^{(0)}\left[C_{i j \ell}\right]_{\kappa \lambda}\right] .
\end{aligned}
$$

The integral has the form in terms of $A_{i j}$ and $J_{i}$; then we get the expression

$$
\begin{aligned}
& \frac{2 i k}{\hbar} \int \prod_{m} d a_{m}^{(0)} \\
& \cdot \exp \left[\frac{i}{\hbar} k\left(\sum_{m} \lambda_{m}\left[\left(a_{m}^{(0)}\right)^{2}+\frac{1}{k} a_{m}^{(0)} \eta_{m}\right]\right)\right] \cdot\left[\frac{3}{8}\right. \\
& \left.\cdot \Theta^{\kappa \lambda} \sum_{i, j, \ell} \lambda_{j} \lambda_{\ell} a_{i}^{0} a_{j}^{0} a_{\ell}^{(0)}\left[C_{i j \ell}\right]_{\kappa \lambda}\right]=\frac{3}{8} \\
& \cdot \Theta^{\kappa \lambda} \sum_{i, j, \ell} \lambda_{j} \lambda_{\ell}\left[C_{i j \ell}\right]_{\kappa \lambda} \exp \left(-\frac{i}{4 \hbar k} \int_{M} \eta \wedge d \eta\right) \\
& \cdot\left[-\frac{i}{4 \hbar k^{2}} \eta_{i} \eta_{j} \eta_{\ell}+\frac{1}{2 k}\left(\frac{\delta_{i \ell} \eta_{j}}{\lambda_{i}}+\frac{\delta_{j \ell} \eta_{i}}{\lambda_{j}}+\frac{\delta_{i j} \eta_{\ell}}{\lambda_{j}}\right)\right] .
\end{aligned}
$$

Thus, the total contribution to the Jones-like polynomial up to first order in $\Theta$ defined in (38) is obtained from the superposition of (39), (53), and (55), yielding to the expression

$$
\begin{aligned}
& J_{C}(\Theta)=\exp \left(-\frac{i}{4 \hbar k} \int_{M} \eta \wedge d \eta\right)[1 \\
& \left.+\frac{3}{16 k} \Theta^{\kappa \lambda} \sum_{i, j}\left(\left[C_{i j i}\right]_{\kappa \lambda} \lambda_{j} \eta_{j}+\left[C_{i j j}\right]_{\kappa \lambda} \lambda_{j} \eta_{i}\right)\right] .
\end{aligned}
$$

As we can see the 0 -th order is the usual $U(1)$ "Jones" polynomial, where $\eta$ is the Poincaré dual of $C$ and the following term is a polynomial over $\Theta$ related to the noncommutativity up to first order.

\section{Noncommutative Aharonov-Bohm Effect}

This section is devoted to explore some physical applications of the noncommutative Wilson loops and linking numbers; in particular we consider the Aharonov-Bohm effect which is a very good arena to test the physical ideas and extract visible effects. We are aware that some noncommutative extensions of the Aharonov-Bohm effect are present in the literature; see, for instance, [42-47]. We will see that our results will agree with their results. Aharonov-Bohm effect consists of an electron beam through a double slit in presence of a small impenetrable solenoid which has a nonvanishing constant magnetic field inside (and therefore a nonvanishing vector potential $A_{\mu}^{(0)}$ ). Outside the solenoid the magnetic field is zero, but not the potential; thus an interference pattern is observed due to the fact that the vector potential is nonvanishing. The effect is measured as a phase factor in the wave function.

In the usual Aharonov-Bohm effect it is assumed that the wave function is of the form $\Phi=\phi \exp (F)$. Under this ansatz one finds the value of $F$ by means of the covariant derivative $D_{j} \exp (F)=k_{j} \exp (F)$. In our case, we will assume 
that the corresponding noncommutative function $\widehat{F}$ can be expanded in terms of the noncommutative parameter $\Theta$; given by the ansatz [45], $\widehat{F}=F^{(0)}+F^{(1)}+F^{(2)}+\cdots$. We will also determine $\widehat{F}$ using the covariant derivative defined in the first section. Thus we have

$$
D_{j} \star \exp _{\star}(\widehat{F})=k_{j} \exp _{\star}(\widehat{F}) ;
$$

considering just the expansion up to second order, it can be written up to second order as

$$
\begin{aligned}
\partial_{j} & {\left[\exp \left(F^{(0)}\right)\left(1+F^{(1)}+F^{(2)}+\frac{1}{2}\left(F^{(1)}\right)^{2}\right)\right] } \\
& -i\left(A_{j}^{(0)}+A_{j}^{(1)}+A_{j}^{(2)}\right) \\
& \cdot \exp \left(F^{(0)}\right)\left(1+F^{(1)}+F^{(2)}+\frac{1}{2}\left(F^{(1)}\right)^{2}\right)+\frac{1}{2} \\
& \cdot \Theta^{\kappa \lambda}\left[\partial_{\kappa}\left(A_{j}^{(0)}+A_{j}^{(1)}\right)\right]\left[\partial_{\lambda}\left(F^{(0)}+F^{(1)}\right)\right]=k_{j} .
\end{aligned}
$$

Thus we obtain the following equations at each order:

$$
\begin{aligned}
& \partial_{j} F^{(0)}-i A_{j}^{(0)}=k_{j}, \\
& \partial_{j} F^{(1)}-i A_{j}^{(1)}+\frac{1}{2} \Theta^{\kappa \lambda}\left(\partial_{\kappa} A_{j}^{(0)}\right)\left(\partial_{\lambda} F^{(0)}\right)=0, \\
& \partial_{j} F^{(2)}-i A_{j}^{(2)}+\frac{1}{2} \Theta^{\kappa \lambda}\left(\partial_{\kappa} A_{j}^{(0)}\right)\left(\partial_{\lambda} F^{(1)}\right) \\
& +\frac{1}{2} \Theta^{\kappa \lambda}\left(\partial_{\kappa} A_{j}^{(1)}\right)\left(\partial_{\lambda} F^{(0)}\right)=0 .
\end{aligned}
$$

We can solve $F^{(0)}$ in terms of $A_{j}^{(0)}$; then we solve for $F^{(1)}$ in terms of $A^{(1)}$ and $F^{(0)}$, and so on. In this way we find explicitly at each order the $F^{(i)}$ 's

$$
\begin{aligned}
F^{(0)}= & k_{j} x^{j}+i \int_{C} A_{j}^{(0)} d x^{j} \\
F^{(1)}= & i \int_{C} A_{j}^{(1)} d x^{j}-\frac{1}{2} \Theta^{\kappa \lambda} \int_{C}\left(\partial_{\kappa} A_{j}^{(0)}\right)\left(\partial_{\lambda} F^{(0)}\right) d x^{j}, \\
F^{(2)}= & i \int_{C} A_{j}^{(2)} d x^{j}-\frac{1}{2} \Theta^{\kappa \lambda} \int_{C}\left(\partial_{\kappa} A_{j}^{(0)}\right)\left(\partial_{\lambda} F^{(1)}\right) d x^{j} \\
& -\frac{1}{2} \Theta^{\kappa \lambda} \int_{C}\left(\partial_{\kappa} A_{j}^{(1)}\right)\left(\partial_{\lambda} F^{(0)}\right) .
\end{aligned}
$$

Finally the expression for $\widehat{F}$ up to second order is given by

$$
\begin{aligned}
\widehat{F} & =k_{j} x^{j}+i \int_{C}\left(A_{j}^{(0)}+A_{j}^{(1)}+A_{j}^{(2)}\right) d x^{j}-\frac{1}{2} \\
& \cdot \Theta^{\kappa \lambda} \int_{C}\left[\left(\partial_{\kappa} A_{j}^{(0)}\right)\left(\partial_{\lambda} F^{(0)}\right)+\left(\partial_{\kappa} A_{j}^{(0)}\right)\left(\partial_{\lambda} F^{(1)}\right)\right. \\
& \left.+\left(\partial_{\kappa} A_{j}^{(1)}\right)\left(\partial_{\lambda} F^{(0)}\right)\right] d x^{j} .
\end{aligned}
$$

From this equation we easily recognize the second term which corresponds to the second order expansion of the noncommutative Wilson loop, and the following terms are the noncommutative corrections to the holonomy.
In the usual Aharonov-Bohm effect the potential outside the solenoid is given by

$$
\begin{aligned}
& A_{1}^{(0)}=-\frac{x_{2}}{x_{1}^{2}+x_{2}^{2}}, \\
& A_{2}^{(0)}=\frac{x_{1}}{x_{1}^{2}+x_{2}^{2}}
\end{aligned}
$$

for reference the expansion up to first and second order of this potential is

$$
\begin{aligned}
& A_{1}^{(1)}=\frac{1}{2} \Theta^{12} \frac{x_{2}}{\left(x_{1}^{2}+x_{2}^{2}\right)^{2}}, \\
& A_{2}^{(1)}=-\frac{1}{2} \Theta^{12} \frac{x_{1}}{\left(x_{1}^{2}+x_{2}^{2}\right)^{2}}, \\
& A_{1}^{(2)}=-\frac{1}{4}\left(\Theta^{12}\right)^{2} \frac{2 x_{2}^{3}+x_{1}^{2} x_{2}}{\left(x_{1}^{2}+x_{2}^{2}\right)^{4}}, \\
& A_{2}^{(2)}=\frac{1}{2}\left(\Theta^{12}\right)^{2} \frac{2 x_{1}^{3}+x_{1} x_{2}^{2}}{\left(x_{1}^{2}+x_{2}^{2}\right)^{4}} .
\end{aligned}
$$

Meanwhile inside the components of the solenoid are

$$
\begin{aligned}
& A_{1}^{(0)}=-\frac{B}{2} x_{2}, \\
& A_{2}^{(0)}=\frac{B}{2} x_{1},
\end{aligned}
$$

where $B$ is the magnitude of a constant magnetic field. At first and second order the potential is given by

$$
\begin{aligned}
& A_{1}^{(1)}=-\frac{3}{8} B^{2} \Theta^{12} x_{2}, \\
& A_{2}^{(1)}=\frac{3}{8} B^{2} \Theta^{12} x_{1}, \\
& A_{1}^{(2)}=-\frac{5}{16} B^{3}\left(\Theta^{12}\right)^{2} x_{2}, \\
& A_{2}^{(2)}=\frac{5}{16} B^{3}\left(\Theta^{12}\right)^{2} x_{1} .
\end{aligned}
$$

In analogy to the usual Aharonov-Bohm effect the phase difference is modified proportional to the flux through the solenoid. Then substituting (64) and (65) into (61) we obtain the wave function's phase of the noncommutative AharonovBohm effect,

$$
\widehat{F}=k_{j} x^{j}+i \pi B r^{2}+i \frac{1}{2} \Theta^{12} \pi B r^{2}+\frac{5}{8} i\left(\Theta^{12}\right)^{2} \pi B r^{2},
$$

where $\Phi=\pi r^{2} B$ is the flux of the magnetic field through the solenoid (whose radius is $r$ ). Finally the correction to the phase due the noncommutativity is

$$
\begin{aligned}
& \exp (\widehat{F}) \approx \exp \left(\frac{i e}{\hbar} k_{j} x^{j}\right) \\
& \cdot \exp \left[\frac{i e}{\hbar} \Phi+\frac{3 i e}{4 \hbar} \Phi \Theta^{12}+\frac{5 i e}{8 \hbar} \Phi\left(\Theta^{12}\right)^{2}\right]
\end{aligned}
$$


As we can see the first term in the imaginary exponential is the standard holonomy (commutative); the second and third terms are corrections to the holonomy due to the noncommutativity up to second order given in terms of the usual flux and the noncommutative parameter.

Now we proceed to show that the phase of (67) is related to the noncommutative Landau levels (see, e.g., [53]). To check closely this affirmation let us make some explicit computations. First of all we define the noncommutative canonical momentum $\widehat{\Pi}_{\mu}$ by minimal coupling changing the usual gauge field $A_{\mu}$ by its noncommutative one $\widehat{A}_{\mu}$; that is,

$$
\widehat{\Pi}_{\mu}=p_{\mu}+e \widehat{A}_{\mu}=p_{\mu}+e\left(A_{\mu}^{(0)}+A_{\mu}^{(1)}+A_{\mu}^{(2)}+\cdots\right) .
$$

In addition, the consideration of quantum mechanical systems in a noncommutative space leads to the following commutation relations:

$$
\begin{aligned}
& {\left[p_{\mu}, p_{\nu}\right]=0,} \\
& {\left[x_{\mu}, x_{\nu}\right]=0,} \\
& {\left[p_{\mu}, x_{\mu}\right]=-i \hbar \delta_{\mu \nu} .}
\end{aligned}
$$

With the aid of previous relations and bearing in mind expressions (64)-(65) let us compute the following commutator up to first order in $\Theta$; this is given by

$$
\left[\widehat{\Pi}_{1}, \widehat{\Pi}_{2}\right]=-i e \hbar B\left(1+\frac{3}{4} \Theta^{12}\right) .
$$

Let us construct the creation-annihilation operators as

$$
\begin{gathered}
a=\frac{\widehat{\Pi}_{1}-i \widehat{\Pi}_{2}}{\sqrt{(2 e \hbar B)\left(1+(3 / 4) \Theta^{12}\right)}}, \\
a^{\dagger}=\frac{\widehat{\Pi}_{1}+i \widehat{\Pi}_{2}}{\sqrt{(2 e \hbar B)\left(1+(3 / 4) \Theta^{12}\right)}},
\end{gathered}
$$

which satisfy the usual relation $\left[a, a^{\dagger}\right]=1$. Now we will consider the hamiltonian $H=(1 / 2 m)\left(\widehat{\Pi}_{1}^{2}+\widehat{\Pi}_{2}^{2}\right)$ and rewrite it in terms of $a$ and $a^{\dagger}$ :

$$
H=\frac{e B \hbar}{2 m}\left(1+\frac{3}{4} \Theta^{12}\right)\left(a a^{\dagger}+a^{\dagger} a\right)
$$

where the factor $\omega=(e B / 2 m)\left(1+(3 / 4) \Theta^{12}\right)$ is the angular frequency. Using the normal ordering we finally get

$$
H=\hbar \omega\left(a^{\dagger} a+\frac{1}{2}\right) \text {. }
$$

By examination of the phase in (67) up to first order, it clearly contains the frequency of the noncommutative Landau levels.

To estimate the order of $\Theta$ we can compare with the results in [45], where they do not use the Seiberg-Witten map. Similar to [45], it is possible to formulate the problem of scattering charged particles in an effective radial potential. The computation is exactly the same. Thus, at the first order we obtain the same result as that in [45] if we make the following substitution: $\Theta$ by $-3 \Theta^{12}$. Then we conclude that the noncommutative parameter is precisely of the same order of magnitude as they had been in [45] estimated $\Theta^{12} \approx$ $[10 \mathrm{Tev}]^{-2}$.

\section{Final Remarks}

In this paper we proposed to use the gauge field provided by the Seiberg-Witten map to study noncommutative Wilson loops. After a brief account on noncommutative Wilson loops, we study abelian Chern-Simons theory on a threedimensional manifold. It was shown that the effect of noncommutativity is the appearance of $6^{n}$ new knots at the $n$th order of the Seiberg-Witten expansion. These knots constitute trivial homology cycles which are Poincaré duals to the highorder Seiberg-Witten potentials of the expansion. Moreover the linking number at $n$th order of a standard 1-cycle with the multiple Poincaré duals of the gauge fields is shown to be written as the sum of the linking number of this 1-cycle with the multiple Poincaré duals of the Seiberg-Witten gauge fields at this order (32). The generalization to higher dimensions can be done straightforwardly.

Furthermore as a topological application of the noncommutative gauge theories and Wilson loops in the abelian case, by using the path integral formalism and the ChernSimons theory we computed the first order and nonvanishing correction due to the noncommutativity of the abelian Joneslike polynomials (56). This term is also of a topological nature and it represents a new noncommutative topological effect of link invariants of three manifolds.

Furthermore as a physical application we compute explicitly the abelian Aharonov-Bohm effect in $\mathbb{R}^{3}$ and calculate the wave function up to second order in the noncommutativity parameter (67). It results in the usual wave function in terms of the eigenvalue $k_{j}$ in the real exponential and in the imaginary part the contribution of the usual flux (commutative) and a second order contribution which is proportional to the square of the flux appear. We also discuss the relation of the Aharonov-Bohm effect and the Landau levels in the noncommutative context. These results are found to agree with those found in [42-47]. In particular, the parameter $\Theta$ is constrained and it basically coincides with that of [45].

It could be interesting to explore some geometrical aspects; we might extend the linking number between knots in the three-dimensional Euclidean space in a noncommutative sense and explore the different orders of the gauge potential and how they could give new information about linking numbers.

Moreover we would like to extend the present noncommutative ideas to higher-dimensional theories, through a $\mathrm{BF}$ theory since it is a higher-dimensional generalization of Chern-Simons theory and the Wilson line will be interpreted in terms of linking numbers between higher-dimensional objects [37-40].

Wilson loops for the spin connection are very important in some theories of quantum gravity [48-51]. A noncommutative version of these models is worth studying by using 
the noncommutative Wilson loops described here. Some of this work is left for future research.

As a further step we are interested in the natural extension to the nonabelian case, where we will deal with two expansions: the first one focusing on the noncommutative parameter and the second one being due to the nonabelianity of the Chern-Simons theory. Also we will study the physical implications using nonabelian Aharonov-Bohm effect [54]. For future work we leave the problem of studying the generalization of noncommutative Aharonov-Bohm effect and its associated Landau levels for this nonabelian case.

\section{Conflict of Interests}

The authors declare that there is no conflict of interests regarding the publication of this paper.

\section{Acknowledgments}

The work of H. García-Compeán was partially supported by the CONACyT research Grant: 128761. The work of O. Obregón is supported by PROMEP, CONACyT, and UG grants. In addition the work of R. Santos-Silva was partially supported by a PROMEP and CONACyT postdoctoral fellowship.

\section{References}

[1] M. R. Douglas and N. A. Nekrasov, "Noncommutative field theory," Reviews of Modern Physics, vol. 73, no. 4, pp. 977-1029, 2001.

[2] R. J. Szabo, "Quantum field theory on noncommutative spaces," Physics Reports, vol. 378, no. 4, pp. 207-299, 2003.

[3] N. Seiberg and E. Witten, "String theory and noncommutative geometry," Journal of High Energy Physics, vol. 1999, no. 9, article 032, 1999.

[4] A. Connes, Noncommutative Geometry, Academic Press, New York, NY, USA, 1994.

[5] B. Jurco, L. Moller, S. Schraml, P. Schupp, and J. Wess, "Construction of non-abelian gauge theories on noncommutative spaces," The European Physical Journal C-Particles and Fields, vol. 21, no. 2, pp. 383-388, 2001.

[6] B. Jurco, L. Moller, S. Schraml, P. Schupp, and J. Wess, "Enveloping algebra-valued gauge transformations for nonabelian gauge groups on non-commutative spaces," The European Physical Journal C-Particles and Fields, vol. 17, no. 3, pp. 521-526, 2000.

[7] P. Aschieri, M. Dimitrijevic, P. Kulish, F. Lizzi, and J. Wess, Noncommutative Spacetimes: Symmetries in Noncommutative Geometry and Field Theory, vol. 774 of Lecture Notes in Physics, Springer, Berlin, Germany, 2009.

[8] X. Calmet, B. Jurčo, P. Schupp, J. Wess, and M. Wohlgenannt, "The standard model on non-commutative space-time," The European Physical Journal C-Particles and Fields, vol. 23, no. 2, pp. 363-376, 2002.

[9] H. Garcia-Compean, O. Obregon, C. Ramirez, and M. Sabido, "Noncommutative self-dual gravity," Physical Review D, vol. 68, no. 4, Article ID 044015, 8 pages, 2003.
[10] H. García-Compeán, O. Obregón, and C. Ramírez, "Gravitational duality in MacDowell-MANsouri gauge theory," Physical Review D, vol. 58, no. 10, Article ID 104012, 1998.

[11] J. A. Nieto, J. Socorro, and O. Obregon, "Gauge theory of supergravity based only on a self-dual spin connection," Physical Review Letters, vol. 76, no. 19, pp. 3482-3485, 1996.

[12] H. García-Compeán, O. Obregón, C. Ramírez, and M. Sabido, "Noncommutative topological theories of gravity" Physical Review D, vol. 68, no. 4, Article ID 045010, 2003.

[13] H. Garcia-Compean, O. Obregon, J. F. Plebanski, and C. Ramirez, "Towards a gravitational analogue to $S$ duality in nonAbelian gauge theories," Physical Review D, vol. 57, article 7501, 1998.

[14] G. 't Hooft, "On the phase transition towards permanent quark confinement," Nuclear Physics B, vol. 138, no. 1, pp. 1-25, 1978.

[15] J. M. Maldacena and J. G. Russo, "Large $N$ limit of noncommutative gauge theories," Journal of High Energy Physics, vol. 1999, no. 9, article 025, 1999.

[16] N. Ishibashi, S. Iso, H. Kawai, and Y. Kitazawa, "Wilson loops in non-commutative Yang-Mills," Nuclear Physics B, vol. 573, no. 1-2, pp. 573-593, 2000.

[17] A. Dhar and Y. Kitazawa, "Wilson loops in strongly coupled noncommutative gauge theories," Physical Review D, vol. 63, no. 12, Article ID 125005, 2001.

[18] W.-H. Huang, "Dual string description of Wilson loop in noncommutative gauge theory," Physics Letters B, vol. 647, no. 5-6, pp. 519-525, 2007, Erratum in: Physics Letters B, vol. 652, p. 388, 2007.

[19] S. Chakraborty, N. Haque, and S. Roy, "Wilson loops in noncommutative Yang-Mills theory using gauge/gravity duality," Nuclear Physics B, vol. 862, no. 3, pp. 650-670, 2012.

[20] A. Dhar and Y. Kitazawa, "Loop equation and Wilson line correlators in non-commutative gauge theories," Nuclear Physics B, vol. 613, no. 1-2, pp. 105-126, 2001.

[21] L. D. Paniak and R. J. Szabo, "Open Wilson lines and group theory of noncommutative Yang-Mills theory in two dimensions," Journal of High Energy Physics, vol. 2003, no. 5, article 29, 2003.

[22] J. Ambjørn, A. Dubin, and Y. Makeenko, "Wilson loops in 2D noncommutative euclidean gauge theory 1 . Perturbative expansion," Journal of High Energy Physics, vol. 2004, no. 7, article 044, 2004.

[23] J. Ambjorn, A. Dubin, and Y. Makeenko, "Wilson loops in 2D noncommutative euclidean gauge theory: 2. 1/ $\theta$ expansion," Journal of High Energy Physics, vol. 2007, no. 10, article 079, 2007.

[24] M. Cirafici, L. Griguolo, D. Seminara, and R. J. Szabo, "Morita duality and noncommutative Wilson loops in two dimensions," Journal of High Energy Physics, vol. 2005, no. 10, article 030, 2005.

[25] M. Riccardi and R. J. Szabo, "Wilson loops and area-preserving diffeomorphisms in twisted noncommutative gauge theory," Physical Review D, vol. 75, no. 12, Article ID 125022, 2007.

[26] W. Bietenholz, A. Bigarini, and A. Torrielli, "Area-preserving diffeomorphisms in gauge theory on a non-commutative plane: a lattice study," Journal of High Energy Physics, vol. 2007, no. 8, article 041, 2007.

[27] N. Nekrasov and A. S. Schwarz, "instantons on noncommutative $\mathbb{R}^{4}$, and $(2,0)$ superconformal six dimensional theory," Communications in Mathematical Physics, vol. 198, no. 3, pp. 689-703, 1998.

[28] P. Aschieri and L. Castellani, "Noncommutative Chern-Simons gauge and gravity theories and their geometric Seiberg-Witten 
map," Journal of High Energy Physics, vol. 2014, no. 11, article 103, 2014.

[29] M. M. Sheikh-Jabbari, "A note on noncommutative ChernSimons theories," Physics Letters B, vol. 510, no. 1-4, pp. 247-254, 2001.

[30] D. J. Gross, A. Hashimoto, and A. N. Itzhaky, "Observables of non-commutative Gauge theories," Advances in Theoretical and Mathematical Physics, vol. 4, p. 893, 2000.

[31] L. Hong, “*-Trek II: ${ }^{*} n$ operations, open Wilson lines and the Seiberg-Witten map," Nuclear Physics B, vol. 614, no. 1-2, pp. 305-329, 2001.

[32] T. Mehen and M. Wise, "Generalized *-products, Wilson lines and the solution of the Seiberg-Witten equations," Journal of High Energy Physics, vol. 2000, no. 12, article 008, 2000.

[33] S. Mukhi and N. V. Suryanarayana, "Open-string actions and noncommutativity beyond the large- $B$ limit," Journal of High Energy Physics, vol. 2002, no. 11, article 002, 2002.

[34] J. A. Harvey, "Topology of the gauge group in noncommutative gauge theory," Proceedings of the strings 2001 meeting, http://arxiv.org/abs/hep-th/0105242.

[35] R. L. Ricca and B. Nipoti, "Gauss' linking number revisited," Journal of Knot Theory and Its Ramifications, vol. 20, no. 10, article 1325, 2011.

[36] E. Witten, "Quantum field theory and the Jones polynomial," Communications in Mathematical Physics, vol. 121, no. 3, pp. 351399, 1989.

[37] G. T. Horowitz, "Exactly soluble diffeomorphism invariant theories," Communications in Mathematical Physics, vol. 125, no. 3, pp. 417-437, 1989.

[38] G. T. Horowitz and M. Srednicki, "A quantum field theoretic description of linking numbers and their generalization," Communications in Mathematical Physics, vol. 130, no. 1, pp. 83-94, 1990.

[39] M. Blau and G. Thompson, "Topological gauge theories of antisymmetric tensor fields," Annals of Physics, vol. 205, no. 1, pp. 130-172, 1991.

[40] H. García-Compeán and R. Santos-Silva, "Link invariants for flows in higher dimensions," Journal of Mathematical Physics, vol. 51, no. 6, Article ID 063506, 2010.

[41] L. Gallot, E. Pilon, and F. Thuillier, "Higher dimensional abelian Chern-Simons theories and their link invariants," Journal of Mathematical Physics, vol. 54, Article ID 022305, 2013.

[42] M. Chaichian, A. Demichev, P. Prešnajder, M. M. SheikhJabbari, and A. Tureanu, "Quantum theories on noncommutative spaces with nontrivial topology: Aharonov-Bohm and Casimir effects," Nuclear Physics B, vol. 611, no. 1-3, pp. 383-402, 2001.

[43] J. Gamboa, M. Loewe, F. Méndez, and J. C. Rojas, "The Landau problem and noncommutative quantum mechanics," Modern Physics Letters A, vol. 16, no. 32, pp. 2075-2078, 2001.

[44] M. Chaichian, P. Prešnajder, M. M. Sheikh-Jabbari, and A. Tureanu, "Aharonov-Bohm effect in noncommutative spaces," Physics Letters B, vol. 527, no. 1-2, pp. 149-154, 2002.

[45] H. Falomir, J. Gamboa, M. Loewe, F. Méndez, and J. C. Rojas, "Testing spatial noncommutativity via the Aharonov-Bohm effect," Physical Review D, vol. 66, no. 4, Article ID 045018, 2002.

[46] K. Li and S. Dulat, "The Aharonov-Bohm effect in noncommutative quantum mechanics," The European Physical Journal CParticles and Fields, vol. 46, no. 3, pp. 825-828, 2006.

[47] S. Dulat and K. Li, "Landau problem in noncommutative quantum mechanics," Chinese Physics C, vol. 32, no. 2, pp. 9295, 2008.
[48] C. Rovelli and L. Smolin, "Knot theory and quantum gravity," Physical Review Letters, vol. 61, no. 10, article 1155, 1988.

[49] A. Ashtekar, "Introduction to loop quantum gravity and cosmology," in Quantum Gravity and Quantum Cosmology, Lecture Notes in Physics, pp. 31-56, Springer, Berlin, Germany, 2013.

[50] E. Witten, “Topology-changing amplitudes in $2+1$ dimensional gravity, Nuclear Physics B, vol. 323, no. 1, pp. 113-140, 1989.

[51] E. Witten, "A note on the Chern-Simons and Kodama wave functions," http://arxiv.org/abs/gr-qc/0306083.

[52] K. Ülker and B. Yapışkan, "Seiberg-Witten maps to all orders," Physical Review D, vol. 77, no. 6, Article ID 065006, 2008.

[53] S. Gangopadhyay, A. Saha, and A. Halder, "On the Landau system in noncommutative phase-space," Physics Letters A, 2015.

[54] P. A. Horváthy, "Nonabelian Aharonov-Bohm effect," Physical Review D, vol. 33, no. 2, pp. 407-414, 1986. 


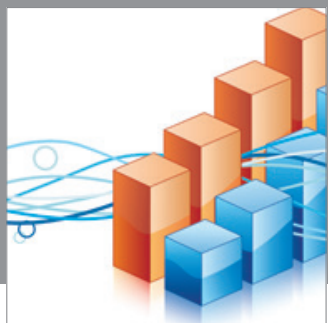

Advances in

Operations Research

mansans

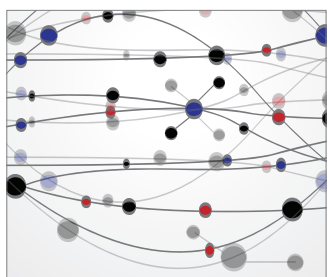

The Scientific World Journal
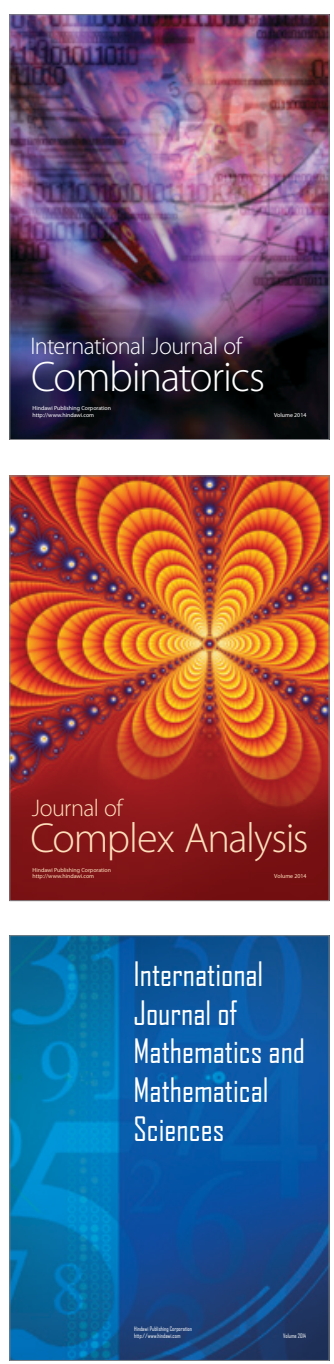
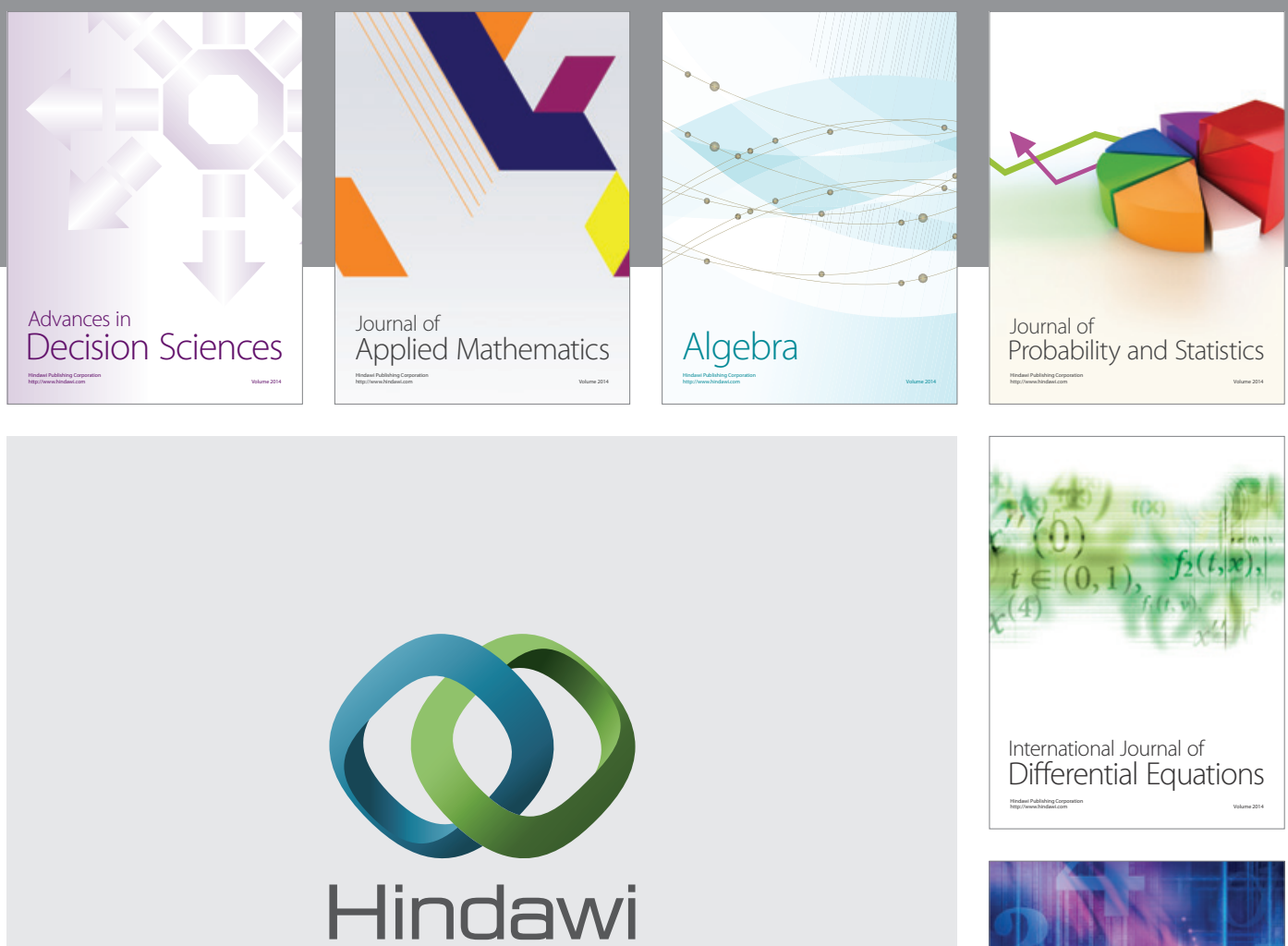

Submit your manuscripts at http://www.hindawi.com
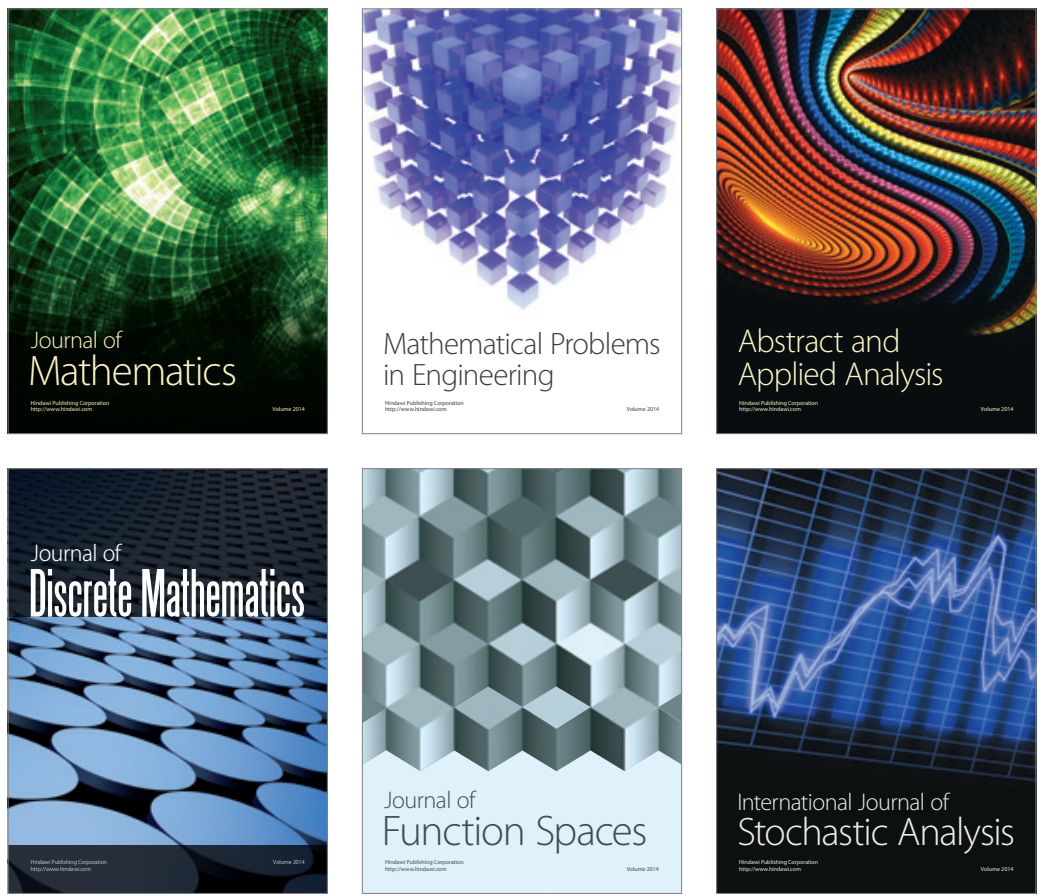

Journal of

Function Spaces

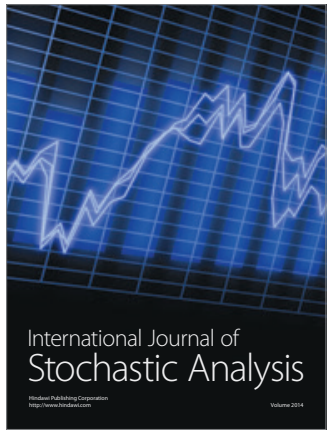

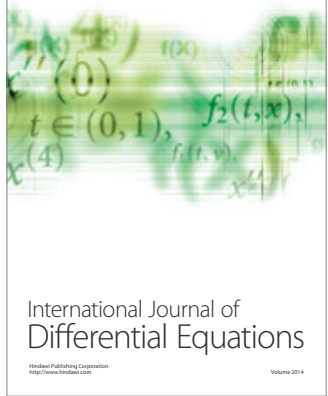
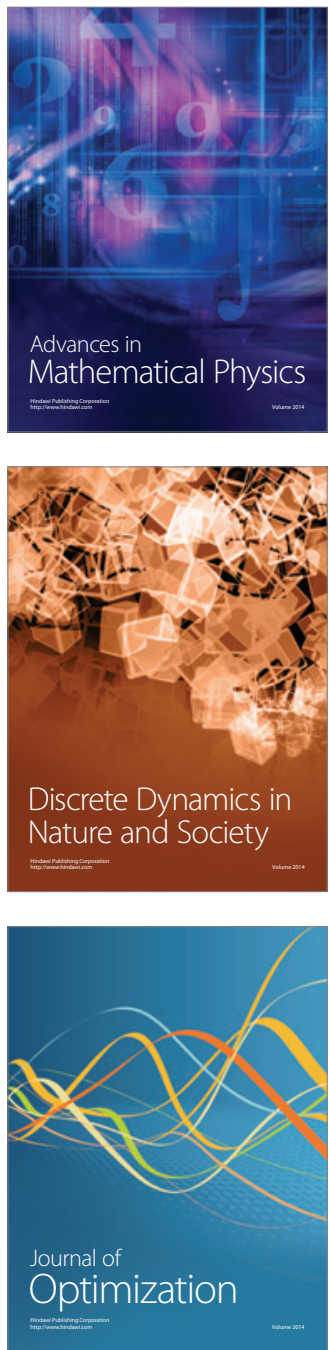